\title{
Prevalence of pathotypes of Phakopsora pachyrhizi Syd. causing Asian soybean rust in India
}

\author{
L. Devaraj, S. Jahagirdar ${ }^{*}$ and G.T. Basavaraja
}

\begin{abstract}
Soybean rust caused by Phakopsora pachyrhizi Syd. is a potential disease causing severe losses in yield and oil quality of soybean [Glycine max (L.) Merr.]. The physiological variability studies were carried out for 25 isolates collected from different growing environment of Karnataka, India during Kharif 2010-2012. Morphological and cultural characters of 25 isolates were studied under identical condition. Among 25 isolates, the maximum uredospore size was found in Ugarkhurd isolate $(90.93 \times 70.05$ $\mu \mathrm{m})$. The differential JS-335 reacted to the maximum of eight isolates, and isolate Rayanal produced both $\mathrm{RB}$ and TAN reaction on most of the differentials. In the clustering of different isolates into pathotypes, the cluster analysis indicated the expression of eight isolates on differential JS-335 and there was no expression of all the isolates on differential PI-459024B. In cluster-II, nine isolates expressed on PI-200492, while one isolate expressed on JS-335. In cluster-III, 23 isolates expressed on PI-459024B and 12 isolates expressed on PI-200492. Hence, this study described the three pathotypes that causes soybean rust in India namely, Cluster I-Reddish Brown (RB) lesion producing pathotypes, Cluster II - TAN lesion producing pathotypes and Cluster III - Mixed or RB + TAN producing pathotypes. This information could further be used for identification of race pattern in Karnataka and other major rust prone areas of India.
\end{abstract}

Keywords: Soybean, Phakopsora pachyrhizi Syd, pathotypes, TAN lesion and RB lesions, differentials, India

\section{Introduction}

Soybean [Glycine max (L.) Merr.] is a widespread crop in the world and ranks first among the oilseed crops. It is a major staple food for the lower strata of the society in India, however, also a crop of par excellence for industrial use. The full potential of the soybean crop is far from being exploited due to several abiotic and biotic stresses. The crop suffers from many fungal diseases and among them foliar diseases take a heavy toll by reducing the yield. Among the foliar diseases, Asian soybean rust caused by Phakopsora pachyrhizi Syd. is the potential disease that causes severe losses in yield and oil quality of soybean. Most of the research on management of soybean rust has focused on screening of genotypes and the use of fungicides. The soybean growers of the Indian subcontinent are seriously affected the rust disease during last two decades with a yield loss ranging from $30-80 \%$ in many parts of India like Karnataka,

\footnotetext{
${ }^{1}$ AICRP on Soybean, University of Agricultural Sciences, Dharwad, Karnataka, India

* Corresponding Author: shamaraoj@gmail.com
} 
Maharashtra, Manipur, Nagaland and Meghalaya. The disease severity depends on the stage of occurrence and favourable climatic conditions (Jahagirdar et al., 2010). Severity of the disease is reported to cause yellowing, premature drying and defoliation. Usually small, yellow lesions appear on the lower leaves in the beginning, which later develops into light brown to dark pustules.

Physiological races of $P$. pachyrhizi also have been reported in Taiwan, Australia, China, and Japan. In Taiwan in 1983, 50 single-urediniospore isolates of $P$. pachyrhizi inoculated onto the five soybean entries namely, plant introduction (PI) 462312 (Ankur), TK-5, TN 4, PI 200492 (Komata), and PI 230971, were differentiated into three physiological races based upon specific reaction patterns of RB and TAN lesions (Yeh, 1983). In Australia, one race was virulent on soybean cv. Williams and avirulent on PI 200492, while another race was virulent on both soybean entries (McLean and Byth, 1977). In another study, six races of $P$. pachyrhizi were identified using 257 entries from six Glycine spp. (Burdon and Speer, 1984). In China, seven P. pachyrhizi isolates were differentiated into four races using a set of eight soybean entries (Tan and Sun, 1989). In Japan, 18 P. pachyrhizi races were differentiated using 11 soybean entries based upon reaction type and the number of uredinia produced per lesion (Yamaoka et al., 2002). However, there is no clear information on pathotypes of $P$. pachyrhizi in the subcontinent. Hence, this study was conducted to understand the prevailing pathotypes based on reaction on 13 international set of differentials.

\section{Materials and Methods}

Different infected samples were collected representing isolates of Phakopsora pachyrhizi of Karnataka. The uredinial collections of $P$. pachyrhizi were made from soybean leaves collected from twelve different locations of northern part of Karnataka (Bagalkot, Belgaum, Bidar, Dharwad and Haveri districts) in India. Collection consisted of one to several leaves bearing uredinia from a single plant or mainly from the cultivar JS 335. The rust spores were harvested immediately from the infected leaves. Spores were tapped and teased out from the pustules with the help of sterilized needle. Uredospores were collected in sterilized two $\mathrm{ml}$ centrifuge tubes, labeled and stored at $-20{ }^{\circ} \mathrm{C}$ in deep freezer for further studies. Prior to inoculation, stored uredospores were removed from deep freezer and heat-shocked at $40{ }^{\circ} \mathrm{C}$ for $5 \mathrm{~min}$. Uredospores were hydrated overnight by floating them in a small centrifuge tubes on sterile distilled water. The inoculum of each isolate was prepared by suspending uredospores in $0.1 \%$ tween 20 (sodium monolaurate) in sterile distilled water, mixing vigorously and filtering through a spore filter cloth. The uredospore concentration was adjusted to 20,000 uredospores/ml for spray inoculation (Devaraj, 2012). The collected isolates were inoculated on different host differentials to study, the variation in P. pachyrhizi isolates, number of lesions, reaction type (nature of 
virulence) and lesion shape on the host under glasshouse condition. Based on these observations pathotype cluster were created for $P$. pachyrhizi isolates representing different geographical areas to know variation on host differentials.

\section{Results and Discussion}

The results of the study are presented in Tables 1 to 3 . The 25 isolates collected showed irregular, circular to ellipsoidal shape of uredospores irrespective of the location of collection. The colour of uredospore ranged from pale brown to dark, orange brown and colourless in some isolates. The size of the uredospore ranged from 62.41 to $90.93 \mu \mathrm{m}$ in length while the width of the uredospores ranged from 43.01 to $70.05 \mu \mathrm{m}$. Two isolates from Mudhol Dharwad and Kundgol, three isolates from Athani and Bailhongal, one isolate each from Chikkodi and Hukkeri taluks exhibited circular and ellipsoidal nature of uredospores. Two isolates from Bailhongal and Kundgol, five isolates from Chikkodi, one each from Hubli and Kalaghatagi exhibited irregular and ellipsoidal nature of uredospores. The Sankeshwar isolate of Hukkeri taluk was more distinct with circular, irregular and ellipsoidal nature of uredospore.

There was a distinct variability in the colour of uredospores among different isolates. Malali and Mudhol isolate from Mudhol taluk showed orange brown colour of uredospores, while Bailhongal isolate from Bailhongal, Aadi, Bivishi, Kallur and Kotabagi from Chikkodi taluk, Yamakaradi from Hukkeri taluk and Dharwad isolate exhibited dark brown coloured uredospores. The Ugarkhurd and Ugar BK isolates from Athani taluk, Gamanagatti isolate from Hubli, Rayanal isolate from Kundgol taluk recorded a pale yellow colour of the uredospores. The Kanavihonnapur isolate of Kalaghatagi taluk, Eliwal isolates from Kundgol Sangankeri cross isolates from Gokak taluk exhibited light pale yellow colour of the uredospores. The isolates from Nayanagar and Hirebagewadi of Bailhongal taluk, Sankeshwar of Hukkeri taluk, Mulamuttla of Dharwad taluk recorded light to dark brown colour of uredospores. The Vaderatti isolate from Bailhongal, Ramankoppa isolate of Kundgol taluk exhibited distinct colour of uredospores ranging from pale brown to colourless. The maximum length $(90.93 \mu \mathrm{m})$ of the uredospores was recorded in Ugarkhurd isolate of Athani taluk followed by $86.95 \mu \mathrm{m}, 81.60 \mu \mathrm{m}$ and $80.61 \mu \mathrm{m}$ in UgarBK, Rayanal and Gamanagatti isolates, respectively. The minimum uredospore length of $62.41 \mu \mathrm{m}$ was recorded in Hirebagewadi isolate of Bailhongal taluk. The maximum width of uredospore was $70.05 \mu \mathrm{m}$ recorded in Ugarkhurd isolate of Athani taluk followed by $66.84 \mu \mathrm{m}$ and $66.30 \mu \mathrm{m}$ in Ugar BK and Rayanal isolates, respectively. The minimum width of $43.01 \mu \mathrm{m}$ was recorded in Kanavihonnapur isolate of Kalaghatagi taluk. 
Table 1. Morphological characters of Phakopsora pachyrhizi isolates from northern Karnataka, India.

\begin{tabular}{|c|c|c|c|c|c|}
\hline \multirow{2}{*}{ Taluk } & \multirow{2}{*}{$\begin{array}{l}\text { Phakopsora pachyrhizi } \\
\text { Isolates }\end{array}$} & \multirow{2}{*}{ Shape of spore } & \multirow{2}{*}{ Color of spore } & \multicolumn{2}{|c|}{ Size $(\mu \mathrm{m})$} \\
\hline & & & & Length & Width \\
\hline \multirow[t]{2}{*}{ Mudhol } & Malali (Ma) & Circular and Ellipsoidal & Orange Brown & 70.13 & 54.21 \\
\hline & Mudhol (Md) & Circular and Ellipsoidal & Orange Brown & 72.42 & 55.27 \\
\hline \multirow[t]{3}{*}{ Athani } & Athani (At) & Circular and Ellipsoidal & Dark Brown & 67.54 & 50.08 \\
\hline & Ugar BK (Ub) & Circular and Ellipsoidal & Pale Yellow & 86.95 & 66.84 \\
\hline & Ugarkhurd (Uk) & Circular and Ellipsoidal & Pale Yellow & 90.93 & 70.05 \\
\hline \multirow[t]{4}{*}{ Bailhongal } & Vaderatti (Va) & Circular and Ellipsoidal & Pale brown to colorless & 72.47 & 59.01 \\
\hline & Bailhongal (Ba) & Irregular and Ellipsoidal & Dark Brown & 75.04 & 51.71 \\
\hline & Nayanagar $(\mathrm{Na})$ & Circular and Ellipsoidal & Light to dark brown & 70.59 & 58.15 \\
\hline & Hirebagewadi $(\mathrm{Hb})$ & Circular and Ellipsoidal & Light to dark brown & 62.41 & 55.43 \\
\hline \multirow[t]{5}{*}{ Chikkodi } & Aadi (Aa) & Irregular and Ellipsoidal & Dark Brown & 70.85 & 50.96 \\
\hline & Bivishi (Bv) & Irregular and Ellipsoidal & Dark Brown & 75.04 & 52.37 \\
\hline & Kallur (Ka) & Circular and Ellipsoidal & Dark Brown & 67.54 & 50.94 \\
\hline & Sirguppi (Si) & Irregular and Ellipsoidal & Light Pale Yellow & 79.05 & 44.54 \\
\hline & Kotabagi (Ko) & Irregular and Ellipsoidal & Dark Brown & 67.92 & 50.78 \\
\hline \multirow[t]{2}{*}{ Hukkeri } & Yamakardi (Ya) & Irregular and Ellipsoidal & Dark Brown & 72.56 & 53.46 \\
\hline & Sankeshwar (Sa) & Circular, Irregular and Ellipsoidal & Light to dark brown & 72.16 & 54.02 \\
\hline \multirow[t]{2}{*}{ Gokak } & Gudasa (Gu) & Irregular and Ellipsoidal & Light Brown & 77.23 & 50.34 \\
\hline & Sangankeri cross (Sc) & Irregular and Ellipsoidal & Light Pale Yellow & 74.53 & 50.95 \\
\hline \multirow[t]{2}{*}{ Dharwad } & Mulmuttla (Mu) & Circular and Ellipsoidal & Light to dark brown & 79.70 & 64.91 \\
\hline & Dharwad (Dh) & Circular and Ellipsoidal & Dark Brown & 67.95 & 53.95 \\
\hline Hubli & Gamangatti (Gt) & Irregular and Ellipsoidal & Pale Yellow & 80.61 & 54.44 \\
\hline Kalaghatagi & Kanavihonnapur Kh) & Irregular and Ellipsoidal & Light Pale Yellow & 75.05 & 43.01 \\
\hline \multirow[t]{3}{*}{ Kundgol } & Eliwal (EI) & Irregular and Ellipsoidal & Light Pale Yellow & 74.53 & 50.95 \\
\hline & Rayanal (Ry) & Circular and Ellipsoidal & Pale Yellow & 81.60 & 66.30 \\
\hline & Ramanakoppa (Ra) & Circular and Ellipsoidal & Pale brown to colorless & 69.38 & 54.20 \\
\hline
\end{tabular}


The maximum size of the uredospore $(90.93 \times 70.05 \mu \mathrm{m})$ was recorded with the Ugarkhurd isolate followed by $86.95 \times 66.84 \mu \mathrm{m}, 81.60 \times 66.30 \mu \mathrm{m}$ and $80.61 \times$ $54.44 \mu \mathrm{m}$ recorded in UgarBK, Rayanal and Gamanagatti isolates, respectively. The minimum size $(62.41 \times 55.43 \mu \mathrm{m})$ of the uredospores was recorded with the Hirebagewadi isolate of Bailhongal taluk. The maximum spore size ( $90.93 \mathrm{X}$ $70.05 \mu \mathrm{m})$ was recorded from the Ugarkhurd isolate and the minimum (62.41 X $55.43 \mu \mathrm{m}$ ) was recorded from the Hirebagewadi isolate. Uredospores are short, vary in the spore colour like hyaline, yellowish brown, orange brown, dark brown, light brown or pale yellow with varying in shape circular, ellipsoidal or irregular as reported by Yang (1977) and Bonde and Brown (1980).

The study revealed that, shape of the spore varied in circular, ellipsoidal and irregular. The colour varied from hyaline to orange brown, light brown to dark brown and light pale yellow to pale yellow were recorded in different isolates. Similar observations were reported on morphological characters of uredia and uredospores, of P. pachyrhizi by Sarbhoy et al. (1972), Yang (1977) and Allen (1983). These variations in size, shape and colour of uredospores largely attributed to pathogenic potential of each isolate. For the first time, we brought interaction strainal variability of P. pachyrhizi in India.

Table 2. Clustering of Phakopsora pachyrhizi based on lesion size and type.

\begin{tabular}{lccc}
\hline \multirow{2}{*}{ Differentials } & \multicolumn{3}{c}{ Pathotypes } \\
\cline { 2 - 4 } & ${\text { Cluster }- \text { I }^{*}}^{\text {Cluster - II }}$ & Cluster - III \\
\hline PI-459024B & 0 & 2 & 23 \\
Pl-459025F & 1 & 4 & 20 \\
EC-241778 & 3 & 8 & 14 \\
EC-241780 & 2 & 2 & 21 \\
EC-391160 & 4 & 6 & 15 \\
PI-230970 & 6 & 3 & 16 \\
PI-200492 & 4 & 9 & 12 \\
JS-335 & 8 & 1 & 16 \\
PI-230971 & 2 & 3 & 20 \\
PI- 459025B & 4 & 6 & 15 \\
EC-462312 & 4 & 4 & 17 \\
TK-5 & 1 & 4 & 20 \\
Wyne & 1 & 6 & 18 \\
\hline
\end{tabular}

${ }^{*}$ Cluster I - Reddish Brown lesion producing pathotypes; Cluster II- TAN lesion producing pathotypes; Cluster III- Mixed lesion(RB \& TAN) producing pathotypes

Upon inoculation to different host differentials, the number of lesions varied from 0 to 8 . The maximum number (8 lesions per leaf) was recorded on differential JS-335 and Pl-459025F for the Sangankeri and Athani isolates. The minimum number of lesions (1) was observed in most of the differentials for different isolates, whereas no lesions were observed for most of the isolates on 
differentials. Reaction type recorded based on the colour of the pustule produced on the leaf viz. reddish brown (RB) colour, tan colour (TAN) and mixed colour (TAN \& RB). The maximum of eight RB on JS-335, nine TAN on PI200492 and most of the reactions showed mixed colour lesions (TAN \& RB) on differentials against different isolates. The observation taken on lesion shape recorded based on the pustule produced on the leaf viz. circular pustule (CP), irregular pustule (IP) and mixed pustules (MP) showed a maximum of eight CP on JS-335, nine IP on Pl-200492, while most of the reaction showed MP on differentials against different isolates.

Based on the above observations, the isolates were categorized into different clusters viz. I (RB lesion producing pathotypes), II (TAN lesion producing pathotypes) and III (Mixed lesion RB \& TAN producing pathotypes). In Cluster-I, RB (Reddish Brown) lesion producing pathotypes were observed, a maximum of eight isolates were recorded on JS-335 followed by six isolates on $\mathrm{PI}-230970$. All the isolates produced RB lesions on all differentials except PI459024B. In cluster-II, TAN lesions producing pathotypes were observed. A maximum of nine isolates showed reaction on $\mathrm{Pl}-200492$ followed by eight isolates on EC-241778, while minimum number (1) of isolate was recorded on JS335. All the isolates produced TAN lesions ranging from 1 to 6 lesions. In clusterIII, grouping with no reaction recorded on the differentials, a maximum of 23 isolates were recorded on differential PI-459024B followed by 21 isolates on EC241780 , while the minimum of 12 isolates were recorded on JS-335.

In the cluster-I, the Mudhol, Bivishi, Sankeshwar, Mulamuttla, Kanavihonnapur, Rayanal, Ramanankoppa and Sangankeri cross isolates formed one group on differential JS-335, followed by Hirebagewadi, Bivishi, Kallur, Kotabagi, Yamakanmaradi and Eliwal forming the other group on $\mathrm{Pl}-230970$. Reaction of the Bivishi isolate was common on EC-241778, EC-241780, EC-391160, $\mathrm{PI}-230970, \mathrm{Pl}-200492, \mathrm{JS}-335$ and TK 5. The Athani isolate was the most distinct with its reaction on $\mathrm{Pl}-459025 \mathrm{~F}$ and EC-462312.

In the cluster-II, differential PI-200492 and EC-241778 showed reaction to the maximum of nine and eight isolates, respectively. The EC-391160, PI-459025B and Wyne differentials recorded reaction to six different isolates individually, while JS-335 recorded only to Ugarkhurd isolate. The differentials PI-459024B showed TAN reaction to Rayanal, Sangankeri Cross isolates while Nayanagar, Rayanal isolates showed on EC-241780 and formed one group. In the cluster-III, except two isolates (Rayanal and Sangankeri Cross), all the 23 isolates formed one group where no reactions were recorded on differential PI-459024B. The differentials Pl-459025F, Pl-230971 and TK-5 showed no reaction for the 20 different isolates individually. The differentials EC-241778 and JS-335 individually recorded no reaction to 14 and 16 different isolates, respectively. 
Table 3. Grouping of different isolates of Phakopsora pachyrhizi in various clusters

\begin{tabular}{|c|c|c|c|c|c|c|}
\hline \multirow{3}{*}{ Differentials } & \multicolumn{6}{|c|}{ Pathotype cluster } \\
\hline & \multicolumn{2}{|r|}{ Cluster - I } & \multicolumn{2}{|r|}{ Cluster - II } & \multicolumn{2}{|r|}{ Cluster - III } \\
\hline & $\begin{array}{l}\text { No. of } \\
\text { isolates }\end{array}$ & Name of isolates & $\begin{array}{l}\text { No. of } \\
\text { isolates }\end{array}$ & Name of isolates & $\begin{array}{l}\text { No. of } \\
\text { isolates }\end{array}$ & Name of isolates \\
\hline $\mathrm{PI}-459024 \mathrm{~B}$ & 0 & - & 2 & Ry, Sc, & 23 & $\begin{array}{l}\text { Ma, Md, At, Uk, Ub, Va, Ba, Gu, Na, Hb, Aa, Bv, Ka, Si, Ko, Ya, } \\
\text { Sa, Mu, Dh, Gt, Kh, El, Ra }\end{array}$ \\
\hline $\mathrm{Pl}-459025 \mathrm{~F}$ & 1 & At & 4 & $\mathrm{Hb}, \mathrm{Ko}, \mathrm{Gt}, \mathrm{Ry}$ & 20 & $\begin{array}{l}\text { Ma, Md, Uk, Ub, Va, Ba, Gu, Na, Aa, Bv, Ka, Si, Ya. Sa, Mu, Dh, } \\
\text { Kh, El, Ra, Sc }\end{array}$ \\
\hline$E C-241778$ & 3 & Uk, Bi, Dh & 8 & $\begin{array}{l}\text { Ba, Ko, Mu, Kh, El, Ry, } \\
\text { Ra, Sc }\end{array}$ & 14 & Ma, Md, At, Ub, Va, Gu, Na, Aa, Bv, Ka, Si, Ya, Sa, Mu, \\
\hline EC-241780 & 2 & $\mathrm{Bv}, \mathrm{Kh}$ & 2 & $\mathrm{Na}, \mathrm{Ry}$ & 21 & $\begin{array}{l}\text { Ma, Md, At, Uk, Ub, Va, Ba, Gu, Hb, Aa, Ka, Si, Ko, Ya. Sa, Mu, } \\
\text { Dh, Gt, El, Ra, Sc }\end{array}$ \\
\hline$E C-391160$ & 4 & $\mathrm{Bv}, \mathrm{Ka}, \mathrm{El}, \mathrm{Sc}$ & 6 & $\mathrm{Na}, \mathrm{Aa}, \mathrm{Ko}, \mathrm{Sa}, \mathrm{Gt}, \mathrm{Kh}$, & 15 & Ma, Md, At, Uk, Ub, Va, Ba, Gu, Hb, Si, Ya, Mu, Dh, Ra, Ra \\
\hline $\mathrm{PI}-230970$ & 6 & $\mathrm{Hb}, \mathrm{Bv}, \mathrm{Ka}, \mathrm{Ko}, \mathrm{Ya}, \mathrm{El}$ & 3 & Sa, Dh, Ry, & 16 & Ma, Md, At, Uk, Ub, Va, Ba, Gu, Na, Aa, Si, Mu, Gt, Kh, Ra, Sc, \\
\hline $\mathrm{PI}-200492$ & 4 & $\mathrm{Bv}, \mathrm{Sa}, \mathrm{El}, \mathrm{Ry}$ & 9 & $\begin{array}{l}\text { At, Uk, Ba, Aa, Ya, Mu, } \\
\text { Gt, Kh, Sc, }\end{array}$ & 12 & Ma, Md, Ub, Va, Gu, Na, Hb, Ka, Si, Ko, Dh, Ra, \\
\hline JS-335 & 8 & $\begin{array}{l}\text { Md, Bv, Sa, Mu, Kh, } \\
\text { Ra, Ry, Sc, }\end{array}$ & 1 & Uk, & 14 & Ma, At, Ub, Va, Ba, Gu, Na, Hb, Aa, Ka, Si, Ko, Ya, Dh, Gt, El, \\
\hline $\mathrm{Pl}-230971$ & 2 & $\mathrm{Na}, \mathrm{Ry}$ & 3 & At, Aa, Sc, & 20 & $\begin{array}{l}\text { Ma, Md, Uk, Ub, Va, Ba, Gu, Hb, Bv, Ka, Si, Ko, Ya, Sa, Mu, Dh, } \\
\text { Gt, Kh, El, Ra, }\end{array}$ \\
\hline $\mathrm{Pl}-459025 \mathrm{~B}$ & 4 & $\mathrm{Aa}, \mathrm{Ka}, \mathrm{Si}, \mathrm{Ry}$, & 6 & Ma, Ko, Sa, El, Ra, Sc, & 15 & $\begin{array}{l}\text { Md, At, Uk, Ub, Va, Ba, Gu, Na, Hb, Bv, Ya, Mu, Dh, Gt, Kh, Ra, } \\
\text { Sc, }\end{array}$ \\
\hline$E C-462312$ & 4 & At, Ka, Ra, Sc, & 4 & Uk, Hb, Ko, Ya, & 17 & $\begin{array}{l}\text { Ma, Md, Ub, Va, Ba, Gu, Na, Aa, Bv, Si, Sa, Mu, Dh, Gt, Kh, El, } \\
\text { Ry, }\end{array}$ \\
\hline TK-5 & 1 & $\mathrm{Bv}$, & 4 & Ma, Uk, Ya, Sc, & 20 & $\begin{array}{l}\text { Md, At, Ub, Va, Ba, Gu, Na, Hb, Aa, Ka, Si, Ko, Sa, Mu, Dh, Gt, } \\
\text { Kh, El, Ry, Ra, }\end{array}$ \\
\hline Wyne & 1 & Ka, & 6 & At, Va, Sa, Gt, El, Sc, & 18 & $\begin{array}{l}\text { Ma, Md, Uk, Ub, Ba, Gu, Na, Hb, Aa, Bv, Si, Ko, Ya, Mu, Dh, } \\
\text { Kh, Ry, Ra, }\end{array}$ \\
\hline
\end{tabular}

"Ma - Malali; Md - Mudhol; At - Athani; Uk - Ugarkhurd; Ub - Ugar BK; Va - Vaderatti' Ba - Bailhongal' Gu - Gudasa; Na - Nayanagar; Hb - Hirebagewadi; Aa - Aadi; Bv - Bivishi; Ka - Kallur; Si - Sirguppi; Ko - Kotabagi; Ya - Yamakardi; Sa - Sankeshwar; Mu - Mulmuttla; Dh - Dharwad; Gt - Gamangatti; Kh Kanavihonnpur; El - Eliwal; Ry - Rayanal; Ra - Ramanakoppa; Sc - Sangankeri cross 
The maximum of eight isolates on JS-335 and the minimum of zero on PI$459024 \mathrm{~B}$ were grouped in cluster-I, nine isolates on $\mathrm{PI}-200492$ and one isolate on JS-335 grouped in cluster-II, and 23 isolates on PI-459024B and 12 isolates on $\mathrm{PI}-200492$ grouped in cluster-III. Similar finding were reported by Yamaokal et al. (2002), Twizeyimana et al. (2009), Pham et al. (2009), Twizeyimana and Hartman (2012) and Maphosa et al. (2013) in the case of soybean rust. The clustering of different isolates based on morphological traits clearly indicated the presence of three pathotypes under Indian conditions. This information will help in breeding for resistance based on location specific pathotype and pyramiding of genes to evolve long term durable resistance. This will definitely pave theway for developing an effective epidemiology and sound forewarning system against rust.

\section{References}

Allen, D.J. (1983): The Pathology of Tropical Food Legumes, John Wiley and Sons, New York. pp. 136-137.

Bonde, M.R. and Brown, M.F. (1980): Morphological comparison of isolates of Phakopsora pachyrhizi from different areas of the world. Canadian Journal of Microbiology., 26: 1443-1449.

Burdon, J.J., and Speer, S.S. (1984): A set of differential Glycine hosts for the identification of races of Phakopsora pachyrhizi Syd. Euphytica, 33: 891-896.

Devaraj, L. (2012): Studies on variability, epidemiology and management of soybean rust in northern Karnataka. M.Sc. Thesis, Uni. Agric. Sci., Dharwad, Karnataka (India).8opp.

Jahagirdar S, Patil, P. V., Patil, R. H., Burhanuddin B. and Vyas, B.N. (2010): Integrated management of Asian soybean rust caused by Phakopsora pachyrhizi in India. International Journal of Plant Protextion, 3(2): 182-185.

Maphosa, M., Talwana, H. and Phinehas, T. (2013): Assessment of comparative virulence and resistance in soybean using field isolates of soybean rust. Journal of Agriculture Sciences, 5(5): 249-257.

McLean, R.J., and Byth, D.E. (1977): Resistance of soybean to rust in Australia. Pages 5861 in: Rust of Soybean-the Problem and Research Needs. INTSOY Ser. No. 12. (Eds: R.E. Ford and J.B. Sinclair). University of Illinois, Urbana.

Pham, T.A., Miles, M.R., Frederick, R.D., Hill, C.B. and Hartman, G.L. (2009): Differential responses of resistant soybean entries to isolates of Phakopsora pachyrhizi. Plant Diseases, 93: 224-228.

Sarbhoy, A., Thapliyal, P.N. and Payak, M.M. (1972): Phakopsora pachyrhizi on soybean in India. Science and Culture, 38: 198.

Tan, Y.J. and Sun, Y.L. (1989): Preliminary studies on physiological races of soybean rust. Soybean Sci. (China) 8:71-74.

Twizeyimana, M. and Hartman, G.L. (2012): Pathogenic Variation of Phakopsora pachyrhizi Isolates on Soybean in the United States from 2006 to 2009. Plant Diseases, 96: 75-81.

Yamaoka, Y., Fujiwara, Y., Kakishima, M., Katsuya, K., Yamada, K., and Hagiwara, H. (2002): Pathogenic races of Phakopsora pachyrhizi on soybean and wild 
host plants collected in Japan Journal of Genetics and Plant Pathology, 68:52-56.

Yeh, C.C. (1983): Physiological Races of Phakopsora pachyrhizi in Taiwan. J. Agric. Res. Chaina, 32 (1): 69-74.

Yang, C.Y. (1977): Soybean rust in Eastern Hemisphere. In: Rust of Soybean - The Problems and Research Needs (Eds. R.E. Ford and J.B. Sinclair), pp 22-23, University of Illinois, USA. 
Deveraj et al. 